\title{
AUTOCUIDADO DE LAS ADULTAS MAYORES CON DIABETES MELLITUS INSCRITAS EN EL PROGRAMA DE ENFERMEDADES CRÓNICAS EN TEMOAYA, MÉXICO
}

\author{
SELF-CARE OF THE OLDER ADULT WITH TYPE 2 DIABETES \\ MELLITUS: NEW RECRUITS TO THE CHRONIC DISEASE PROGRAM \\ IN TEMOAYA, MÉXICO
}

\author{
Vianey MÉndez Salazar ${ }^{*}$ \\ Verónica Becerril Estrada ${ }^{* *}$ \\ Matiana Morales del Pilar ${ }^{* * *}$ \\ Víctor Manuel Pérez Ilagor ${ }^{* * * *}$
}

\begin{abstract}
RESUMEN
Los sujetos de estudio fueron un total de diez adultas mayores con un promedio de 63 años, en la unidad de Salud de San Pedro Arriba, Municipio de Temoaya. La unidad de salud constituye un escenario para la capacitación del usuario, sin embargo se presentan condicionantes en las adultas mayores para no aplicar lo aprendido. El objetivo de la investigación fue el describir las actividades de autocuidado en el adulto mayor con diabetes mellitus tipo 2, inscritos al programa de enfermedades crónicas degenerativas. Se presenta un reporte de investigación de corte cualitativo, se utilizo el método etnográfico, el cual aporta el punto de vista narrativo de la realidad, a partir de las diferentes formas de indagación etnográfica. Para acceder a la información se recurrió a la entrevista a profundidad. La información se registró haciendo énfasis en aquellos que describieron las rutinas y situaciones problemáticas que dio significado a su vida, en el Centro de Salud Rural Disperso. Las acciones que llevan a cabo los adultos mayores son: ejercicio, cuidado de la boca, dieta, monitoreo y cuidado de sus extremidades inferiores en un contexto propio donde existen un conjunto de valores, creencias, tradiciones, símbolos, lenguaje y organización social significativos para los miembros de un grupo, en este caso de los adultos mayores.
\end{abstract}

Palabras clave: Diabetes mellitus tipo 2, adulto mayor, autocuidado.

\begin{abstract}
The subjects of study were ten older adults averaging 63 years of age from the San Pedro Arriba Health Unit in the Municipality of Temoaya. The Health Unit is the location for patient teaching however the reality is that the older adults do not to apply what they learned in the training. The objective of the study was to describe the self-care activities of older adults with type 2 diabetes mellitus registered in the chronic disease program. A qualitative research methodology, ethnography with narrative analysis which differs from other types of ethnographic inquiry, was used. Data were gathered using in-depth interviews. The data were recorded with emphasis on descriptions of daily life and challenging situations that gave meaning to life of patients at the rural health center. The actions that the older adults carry out are: exercise, mouth care, diet, monitoring and care of their low extremities. Findings show that an important set of values, beliefs, traditions, symbols, language and social organization exist for members of this group of older adults.
\end{abstract}

Key words: Type 2 diabetes mellitus, elderly, self-care.

Fecha recepción: 08/09/2009 Fecha aceptación: 18/11/2010

\footnotetext{
* Enfermera. Docente de la Facultad de Enfermería y Obstetricia Universidad Autónoma del Estado de México, México. E-mail: vianneyms@usa.net

** Enfermera. Instituto de Salud en el Estado de México, México. E-mail: maty_2402@yahoo.com.br

${ }^{* * *}$ Enfermera. Docente de la Facultad de Enfermería. Universidad Autónoma del Estado de México, México.

E-mail: maty_2402@yahoo.com.br

**** Enfermero. Docente de la Facultad de Enfermería y Obstetricia Universidad Autonoma del Estado de México, México.

E-mail: vianneyms@usa.net
} 


\section{INTRODUCCIÓN}

La OMS considera adultos mayores a las personas de más de 60 años para los que viven en los países en vías de desarrollo y de 65 años a los que viven en países desarrollados. En 1994 la OPS ajustó la edad de 65 y más para considerarlo adulto mayor. Según el Instituto Nacional de Estadística, Geografía e Informática en México los adultos mayores suman 6,9 millones de habitantes, representando el 7,3\% del total de la población y se estima que en 25 años el sector de adultos mayores pase de 6 a 15,6 millones de personas. $\mathrm{Al}$ respecto existen grandes desafíos para el personal de salud en cuanto a prevención de padecimientos asociados a una larga vida como la diabetes. A medida que envejecen las personas en el área emocional y social, surgen cambios como depresión, aislamiento, maltrato, lo que induce a una baja autoestima que ocasiona descuido en su higiene personal, desinterés por compartir y convivir con compañeros de su edad, y enfermedades como la diabetes tipo 2 (1) que representa una de las primeras causas de mortalidad en nuestro país. Uno de los principales peligros de la diabetes es su avance silencioso, ya que puede ser asintomático y cursar periodos muy variables en cualquier etapa de la vida; es aquí donde se ve afectado su funcionamiento fisiológico, social, con la aparición de situaciones emocionales, lo cual no ha sido identificado para el previo cuidado o atención (2).

El diagnóstico de una enfermedad crónica puede producir estados de ansiedad en el adulto mayor, por ello es importante la consejería de la enfermera para enseñarle al paciente los cuidados que debe llevar a cabo para evitar complicaciones y concienciarlo de la importancia de ser perseverante ante un padecimiento crónico como la diabetes.
He aquí la importancia de la participación del profesional de enfermería en la salud comunitaria, hacia la prevención primaria para evitar llegar a complicaciones; haciendo énfasis en enseñar, educar, motivar niveles de responsabilidades de acuerdo a sus necesidades y capacidades funcionales en cada uno de los adultos mayores. Los servicios de salud son un espacio privilegiado para dar respuesta a las necesidades de los adultos mayores, como el permanecer integrado a la familia, participar en forma activa en su entorno y tomar decisiones para el bienestar de su salud a través del autocuidado (3).

La Secretaría de Salud promueve programas educativos en atención primaria a la salud; uno de ellos es el Paquete Básico de Servicios de Salud, con el lema "de alto impacto a un bajo costo", donde intervienen acciones de prevención y control de la diabetes mellitus, el cual contempla el programa del adulto mayor, con sus respectivas líneas de acción (4).

En el Centro de Salud Rural Disperso en San Pedro Arriba, municipio de Temoaya, se llevan a cabo actividades de autocuidado para el adulto mayor con diabetes mellitus como: detección y control de la diabetes e hipertensión arterial, índice de masa corporal para identificar: peso ideal, sobrepeso y obesidad, revisión de agudeza visual y auditiva, revisión de reflejos en tendones, aplicación de toxoide tetánico y vacuna contra la influenza. Asimismo se brinda orientación psicológica y temas que hacen referencia al autocuidado del adulto mayor (5).

Sin embargo existen condicionantes que impiden al adulto mayor llevar a cabo su autocuidado, entre éstas se pueden citar: condicionantes sociodemográficas, capacidades físicas y emocionales, ingresos económicos insuficientes, roles de género tradicionales que persisten, falta de apoyo familiar, carencia de relaciones afectivas, viudez, desmotivación, acceso a los servicios de salud. 


\section{MATERIAL Y MÉTODO}

La presente investigación se estructuró utilizando un método etnográfico a partir de un enfoque cualitativo. Según Stake, el enfoque cualitativo es holístico, empírico, interpretativo y empático (6). Este enfoque permite estudiar la realidad de un contexto específico en el cual se hizo partícipe al sujeto, en este caso al adulto mayor con diabetes mellitus tipo 2. El estudio fue de tipo descriptivo, porque se representa al sujeto en un contexto determinado. Para Aguirre Baztlán, el método etnográfico: describe la cultura de una comunidad, o de algunos de sus aspectos fundamentales, bajo la perspectiva de comprensión global de la misma (7).

El interés de la presente investigación fue describir las actividades de autocuidado en el adulto mayor con diabetes mellitus tipo 2, inscritos al programa de enfermedades crónicas degenerativas. Para acceder al conocimiento se utilizó la entrevista a profundidad, obteniendo información del propio lenguaje de los sujetos. Siguiendo una selección deliberada e intencional, los sujetos de estudio fueron un total de 10 adultas mayores con un diagnóstico de diabetes mellitus tipo 2 . La selección deliberada consistió en elegir a usuarias que asistieron con frecuencia a las sesiones impartidas por enfermería sobre los cuidados que deben tener para evitar complicaciones debido a su padecimiento. Cabe mencionar que el grupo está conformado por 10 mujeres y 5 hombres, los varones quedaron excluidos por sus inasistencias a las sesiones. Nos interesaba saber qué acciones de autocuidado en la esfera física, psicológica y social llevan a cabo las usuarias para verificar qué tanto aplican estas acciones en su vida cotidiana. La entrevista se llevó a cabo bajo el consentimiento informado (8). Cabe señalar una de las limitantes que se tuvo en las entrevistas, porque las usuarias hablan poco español, por lo que se tuvo que ser muy reiterati- vo con las preguntas y la transcripción de las entrevistas. Posterior a la realización de las entrevistas, éstas se transcribieron, leyeron y examinaron en varias ocasiones para conocer los contenidos y delimitar las categorías; éstas resumen datos empíricos, siendo el significado y sus formas los que permitieron interpretar el fenómeno investigado.

\section{RESULTADOS}

Las adultas mayores poseen ciertas particularidades que las ubican en un contexto geográfico determinado por su dialecto, vestuario, costumbres, la edad y la forma en cómo expresaron sus comentarios. Es interesante mencionar la influencia de sus costumbres y creencias que en determinado momento alteran la dinámica del programa que se imparte en el Centro de Salud. El autocuidado comprende:

\section{Ejercicio}

Los comensales portan chincuete, pantalón de manta, sombrero, camisa de algodón; las mujeres se niegan a ponerse 'pants', prefieren portar su falda tradicional, al respecto comentan:

"La verdad como yo le digo al doctor es que a mi no me gusta el pans, yo lo hago solita en mi casa", "voy a decir la verdad llego mi hijo y le di de comer ya cuando me acordé ya había pasado la hora y no fui a los ejercicio"(Sic) E5, "pues no más se sale mi nuera y yo me meto a mi cuarto, pues cierro la puerta y ahí lo estoy haciendo lo ejercicio"(Sic) E10. Cabe señalar que no todas las titulares acuden a realizar ejercicios por varias razones: el traslado al centro de salud que está retirado de la comunidad a donde viven, por falta de motivación; el ejercicio es una actividad ajena a su cotidianidad, por ello algunas señoras realizan sus ejercicios cuando no las ven; otro as- 
pecto son las diversas ocupaciones en el hogar, como atención de los hijos y nietos entre otras, que hacen que la asistencia al Centro de Salud pase a segundo término.

\section{Alimentación}

Su alimentación en domingo es de algunos productos que han caracterizado al tianguis; como barbacoa de carnero, chicharrón de cerdo, carnitas, longaniza, carpa, tamales de charal y otros productos con los que se prepara el tradicional taco de plaza, desde acociles, aguacate, pata de res, pápalo y demás hierbas comestibles. La comida se acompaña de bebidas como agua simple o de frutas, refrescos embotellados, cerveza o pulque.

En el Centro de Salud se les recomienda a los adultos mayores consumir alimentos de la región como quintoniles, quelites, flores de calabaza, calabaza, chayote, tortillas con moderación y fruta de temporada. Sobre la alimentación comentan las entrevistadas:

"Somos provinciano pues la verdad no hay trabajo para ganar un centavo le digo no hay trabajo, si sobra el dinero compro un kilo de retazo, pero no vas a decir que a diario comemo carne, frijol poquito o haba"(Sic) E9, "la doctora dice que ya no coma azúcar ni toma grasa dice"(Sic) E2, "como a las dos de la tarde, a veces un kilo de frijolito o medio kilo de arroz, pollo verduras, a las 8 de la noche un pedazo de tostado y leche"(Sic) E5.

Es difícil llevar una dieta balanceada y mantener en equilibrio los niveles de glucosa, colesterol y urea, cuando los ingresos económicos dependen en gran medida de la venta de animales domésticos, de tejidos y hortalizas. Respecto a la alimentación, las adultas mayores han comprendido en gran medida la importancia de disminuir el consumo de azúcar, la ingesta de proteínas y grasas está sujeta a sus escasos ingresos.

\section{Salud bucal}

En el programa de enfermedades crónico- degenerativas se le enseña al adulto mayor la técnica de cepillado de dientes, se les pide que traigan pasta dental, cepillo de dientes, espejo y un vaso o recipiente con agua. Primero la enfermera explica la técnica del cepillado, este procedimiento les causa angustia, al ver que existe perdida de piezas dentarias y que al momento de cepillarse lo notan, sin embargo en otro momento la pérdida de piezas dentarias lo veían como algo natural. Varias de las entrevistadas manifestaron tener problemas dentales: "Me duele mucho mi diente" E10, "ya no tengo muelas, solo los de enfrente". "Se cayeron solitos" E9. La enseñanza sobre el autocuidado de la boca les ha permitido a las pacientes darle importancia a la higiene bucal, siendo una labor de convencimiento y concienciación del personal de enfermería hacia las adultas mayores. Cuando se les preguntó a las pacientes si se cepillaban sus dientes, la mayoría contestó que sí lo realizaban, que en el centro de salud les habían enseñado cómo lavarse adecuadamente sus dientes: "lo lavo de mañana y de tarde con la crema" E10, "no como dulces ni chocolates" E5, "ahora que me enseñaron lo lavo con Colgate" E9. La participación dentro del club de personas con diabetes y su contacto con el médico y el equipo le permitirá contemplar su educación a lo largo del tiempo. Una cuestión básica de la educación es darle las herramientas para que el adulto mayor pueda aprender por sí mismo, a través de autoaprendizaje aprendieron a cepillarse los dientes adecuadamente; a pesar de hacer énfasis en el cuidado de sus dientes, en las entrevistas ellas manifestaron no cepillarse con regularidad y no tener dinero para comprar una pasta dental.

\section{Vestido y calzado}

Es relevante para los adultos mayores de San Pedro Arriba portar su vestimenta tradicional que los hace sentirse seguros y les brinda identidad para llevar a cabo las actividades cotidianas en su contexto. El vestuario consta de faja chincuete, blusa de colores fuertes, 
los hombres usan ropa de manta, huaraches, cinturón, gabán de algodón y sombrero: "Hago ejercicio con mi chincuete, zapato y mi sombrero" E7, "para el ejercicio no me pongo pantalón nada más mi puro chincuete" E10. El uso de huaraches les puede ocasionar lesiones en los pies siendo más sensibles a lesiones e infecciones los pacientes con diabetes, entre el 40\% y el 70\% de amputaciones en pie se producen por causas relacionadas con la diabetes (8).

Por ello las enfermeras del centro de salud les hacen hincapié a las pacientes de seguir los seis pasos para tener los pies sanos, como: 1. revisar diariamente que sus pies no presenten cortadas, contusiones, arañazos, heridas, decoloración, enrojecimiento, callos e infecciones; 2. Lavar y secar los pies, colocar crema sobre la piel reseca para evitar que se parta, cortarse las uñas con mucho cuidado, 3. Utilizar zapatos cerrados con la medida adecuada. 4. No comprar zapatos apretados 5. Usar calcetines limpios de algodón y lana. 6. Si tienen callos acudir con un profesional (9).

La exploración de extremidades inferiores es importante para el cuidado de los pies. La finalidad es que la usuaria conozca la sensibilidad ante cambios bruscos de temperatura de frío a caliente, así como evitar la aparición de micosis y callosidades con el paso del tiempo. El calzado es determinante para tomar la posición correcta de la persona, así como la comodidad de evitar lesiones que pueden provocar dolor hasta llegar a una luxación e inclusive la pérdida de una extremidad inferior como amputación. Por lo anterior mencionado se les recomienda a los pacientes el autocuidado de sus pies: " $y o$ nunca he cortado los dedo ni los pies yo porque ya me dijo el doctor Acevedo cuídese porque si no los que tienen diabetes se puede infectar puede tener gangrena" (Sic) E1. Al realizar exploración de los pies se encontró que un 50\% de las usuarias manifiestan lesiones, es decir callosidades, micosis, mal olor. Esto se debe a su condición precaria y al uso de calzado de tela, plástico y huaraches de piel, este último es de uso tradicional.

\section{Automonitoreo}

En la clínica se revisan los niveles de glucosa en ayunas, en forma programada, también se toma la presión arterial, circunferencia abdominal, revisión de oídos y agudeza visual. El trabajador en atención primaria a la salud (TAP) revisa el esquema de vacunación y aplica vacunas en caso de necesitarlas. Asimismo se les informa de la funcionalidad de las vacunas y se registran en la cartilla de vacunación del adulto mayor. Además de tener el carnet, cartilla y expediente, la mayoría cuenta con una ficha de identificación, que porta cada vez que va a la clínica de salud. Estos documentos los deberá llevar en cada visita al Centro de Salud: "El marte vengo para que me cheque el doctor porque los oídos me rezumban mucho" (Sic) E7. En caso de inasistencia en un mes, se le informa a la TAP para que realice una visita domiciliaria para darle seguimiento a la usuaria.

\section{Medicamentos}

Según la OPS el adulto mayor consume el doble de medicamentos que una persona joven (10), por tal razón es importante obtener una historia medicamentosa, donde se incluyan alergias, reacciones adversas, fármacos autoprescritos, cuestionarios sobre adicciones como alcohol, tabaco, cafeína. Algunos factores que interfieren con el tratamiento son: nivel socioeconómico, la disminución de agudeza visual, auditiva, de tacto y olfato, de ahí que es sustancial la participación de la familia en el tratamiento del paciente (11).

El significado de los medicamentos para las pacientes entrevistadas es la toma de pastillas que les alivia dolores de cabeza, espalda y zumbidos de oídos: "Tome mi pastilla, el Dr. Gelasio me dijo no la deje tu pastilla" (Sic) E9, 
"El doctor me lo dio una pastilla porque me dolía mucho la cintura" (Sic) E7. "Cuando me duele mi pulmón le pido al señor Ricardo una pastilla y se me quita la dolencia" (Sic) E2.

La orientación que le brinde la enfermera a la paciente es fundamental para evitar en la paciente complicaciones características de su padecimiento, porque el bajo nivel socioeconómico aunado a la edad de las pacientes limita el tratamiento (12).

\section{DISCUSIÓN Y COMENTARIOS}

Los estudios realizados desde un enfoque cultural suelen compartir la definición de cultura como el conjunto de valores, creencias, tradiciones, símbolos, lenguaje y organización social significativa para los miembros de un grupo, en este caso de los adultos mayores. Las aproximaciones sociológicas coinciden en que éste es el resultado de las experiencias vitales, y los acontecimientos sociológicos y culturales se traducen en influencias microambientales que afectan al desarrollo de los individuos. Patrones de crianza de los hijos, presión de grupo, estilos educacionales y oportunidades vocacionales son algunos de los mecanismos a través de los cuales se produce esta influencia. Los valores y normas compartidas en una sociedad proporcionan a los individuos un marco de referencia para guiar sus vidas. Situándonos en el contexto del cuidado, las variables de tipo cultural pueden ejercer su papel en el proceso de estrés del cuidador al enfrentar el deber ser con la realidad de un contexto y un sujeto; en este caso los sujetos de estudio fueron pacientes inscritas en la unidad de salud de San Pedro Arriba, Municipio de Temoaya, en el programa de enfermedades crónicas degenerativas con acciones encaminadas a mejorar la vida de las personas adultas mayores. En el Centro de Salud se les orienta sobre el autocuidado que deben llevar para evitar complicaciones propias de su enfermedad. Una de las actividades que ellos realizan es el ejercitar su cuerpo a través de ejercicios aeróbicos de acuerdo a su edad, condición física y mental. Las adultas mayores realizan los ejercicios utilizando la ropa propia de su región, algunas mujeres se niegan a ponerse tenis y 'pants' porque les es más cómodo usar huaraches, en cuanto al pantalón este tiene un significado para los otomíes, pues sólo lo portan los hombres. Respecto al cuidado bucal, las pacientes no se cepillan los dientes, porque se enrolan en sus quehaceres y no lo llevan a cabo, por lo que es urgente un seguimiento en este aspecto. Otro elemento que se debe tomar en cuenta es el concienciar a las usuarias a usar calzado para evitar lesiones que pueden provocar hiperqueratosis y micosis. En las entrevistas las pacientes manifestaron cuidar sus extremidades colocando pomadas y ellas saben que deben cuidar sus pies para evitar gangrena, sin embargo el uso de huaraches es un riesgo para ellas.

Tocante a la esfera emocional y social, las usuarias refieren que en el Centro de Salud organizan convivios familiares en el centro ceremonial otomí, así como reuniones para celebrar festividades. El hacer partícipe a la familia es de suma importancia para conocer las actividades de prevención que se fomentan en el grupo de ayuda mutua.

La interculturalidad se presenta en los encuentros sociales que se organizan en el centro ceremonial o en las reuniones de grupo en el que se expresan satisfechos, alegres, con ánimo de ocuparse de su persona (13).

Finalmente las acciones que llevan a cabo los adultos mayores son: ejercicio, cuidado de la boca, dieta, monitoreo y cuidado de sus extremidades inferiores en la medida de sus posibilidades, ellas intentan cumplir con las recomendaciones de las enfermeras y los médicos. 


\section{REFERENCIAS}

1. INEGI, Estadística del sector salud y seguridad social, Cuaderno № 20, edición № 5, México; 2004.

2. Alpizar S. Guía para el manejo integral del paciente diabético, $3^{a}$ ed. México: Manual Moderno; 2004.

3. Salgado AA, Guillén FLl, Ruipérez, CI. Manual de Geriatría. 2a ed. España, Salvat: México; 2002.

4. Darth M. Guía Médica y de la Salud para mayores de 50 años, España: Trillas; 1994.

5. Figueroa D. Diabetes, $2^{a}$ ed. España: Masson; 2002.

6. Secretaría de Salubridad y Asistencia. Manual de Paquete Básico de Servicios de Salud, Progresa; 2003.

7. García FE, Musitu OG. Psicología Social de la Familia, 2a ed. Barcelona: Paidós; 2008.

8. Rodríguez G. Metodología de la investigación cualitativa. $2^{a}$ ed. Málaga E. A. Ed. AIJIBE; 1999.

9. Tellez B. Monografía del municipio de Temoaya, México; 2000.

10. Centro Avanzado de tratamiento de heridas difíciles de cicatrizar, úlceras crónicas de extremidades inferiores (n.d.). Hallado en: http://www.portaladultomayor.com/cgibin/pam/display.pl. Acceso el 23 de diciembre de 2008.

11. Aguirre B. Etnografía: Metodología cualitativa en la investigación Sociocultural, 2a ed . México: Alfaomega; 1977.

12. Ruipérez C y B. El paciente anciano. España: Interamericana; 1998.

13. Ley General de Salud, SISTA. México; 2003. 